\title{
Subendometrial Blood Flow is Associated with Pregnancy Outcome of Embryo Transfer in Patients with Thin Endometrium
}

\author{
Yuchen Yan $^{1}$, Jianan Lyu ${ }^{1}$, Qian Zhang ${ }^{2}$, Chunzi Lyu ${ }^{1}$, Meiling Guo ${ }^{1}$, Wei Zhou ${ }^{1}$, Yan Li $^{3}$, \\ and Junhao Yan ${ }^{2}$ \\ ${ }^{1}$ Center for Reproductive Medicine, Cheeloo College of Medicine, Shandong University, \\ Jinan, Shandong \\ ${ }^{2}$ Center for Reproductive Medicine, Shandong University \\ ${ }^{3}$ Affiliation not available
}

February 16, 2022

\begin{abstract}
Objective To assess whether the subendometrial blood flow condition detected by Doppler ultrasound could predict pregnancy outcomes in patients with thin endometrium during in vitro fertilization (IVF) treatment. Design Retrospective cohort study. Setting Reproductive Hospital affiliated with Shandong University, Jinan, Shandong, China Population or Sample The study included 3830 frozen embryo transfer (FET) cycles of 3,830 IVF patients between January 2017 to December 2019. Methods Endometrial thickness, endometrial blood flow, and subendometrial blood flow were measured using transvaginal color Doppler ultrasound, evaluated by experienced clinical ultrasound physicians on the day of endometrial transformation. Logistic binary regression was used to analyze the effects of subendometrial blood flow on successful embryo implantation. Pregnancy outcomes of these patients were documented in clinic medical records during follow-up. Main Outcome Measures Pregnancy outcomes (biochemical pregnancy rate, clinical pregnancy rate and live birth rate) Results A total of 3830 frozen embryo transfer cycles were retrospectively analyzed. Our results show that patients with subendometrial blood flow exhibit higher average success rates of live birth, clinical pregnancy, and embryo implantation compared to those without subendometrial blood flow when endometrial thickness is [?] $0.7 \mathrm{~cm}$. In detail, in thin endometrium patient group, the presence of subendometrial blood flow is correlated with $7.2 \%$ higher biochemical pregnancy rate, $7.0 \%$ higher clinical pregnancy rate, and $6.2 \%$ higher live birth rate compared to those without subendometrial blood flow. Conclusions Endometrial and subendometrial blood flow conditions have predictive value for pregnancy outcomes after frozen embryo transfer in patients with thin endometrium
\end{abstract}

\section{Hosted file}

MS 20220211.docx available at https://authorea.com/users/460738/articles/556619subendometrial-blood-flow-is-associated-with-pregnancy-outcome-of-embryo-transfer-inpatients-with-thin-endometrium 
Figure 1

A

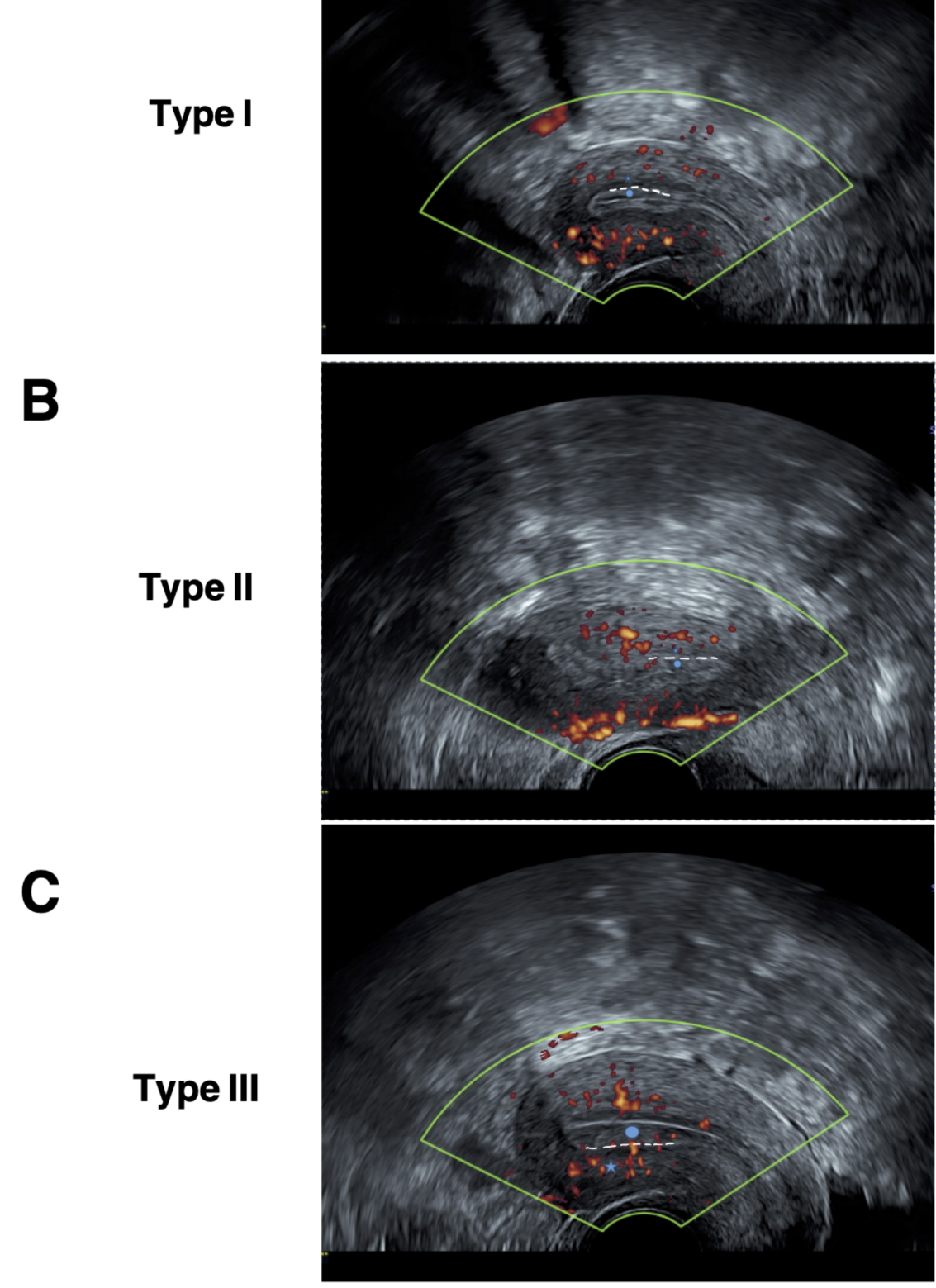

